\title{
Review Article \\ The Ellipsoidal Vortex: A Novel Approach to Geophysical Turbulence
}

\author{
William J. McKiver \\ ISMAR-CNR, Arsenale-Tesa 104, Castello 2737/F, 30122 Venice, Italy \\ Correspondence should be addressed to William J. McKiver; william.mckiver@ve.ismar.cnr.it
}

Received 8 October 2014; Accepted 14 January 2015

Academic Editor: Touvia Miloh

Copyright ( 2015 William J. McKiver. This is an open access article distributed under the Creative Commons Attribution License, which permits unrestricted use, distribution, and reproduction in any medium, provided the original work is properly cited.

\begin{abstract}
We review the development of the ellipsoidal vortex model within the field of geophysical fluid dynamics. This vortex model is built on the classical potential theory of ellipsoids and applies to large-scale fluid flows, such as those found in the atmosphere and oceans, where the dynamics are strongly affected by the Earth's rotation. In this large-scale limit the governing equations reduce to the quasi-geostrophic system, where all the dynamics depends on a single scalar field, the potential vorticity, which is a dynamical marker for vortices. The solution of this system is achieved by the inversion of a Poisson equation, that in the case of an ellipsoidal vortex can be solved exactly. From this ellipsoidal solution equilibria have been determined and their stability properties have been studied. Many studies have shown that this ellipsoidal vortex model, while being conceptually simple, is an extremely powerful tool in eliciting some of the fundamental characteristics of turbulent geophysical flows.
\end{abstract}

\section{Introduction}

In turbulent flow there exist structures whose collective interactions are at the very heart of the dynamics of turbulencevortices. The vortex, a word signifying a type of swirling or spinning fluid, has become a familiar concept in human society, being associated with extreme and highly energetic weather phenomena such as typhoons, tornados, and hurricanes. The relative infrequency of these most dramatic manifestations of vortices could lead one to think that the concept of the vortex itself is limited only to these extreme events, but, on the contrary, vortices are present in almost all fluid phenomena, from the smallest scale flow of water in pipes to the largest scales within the atmosphere and the oceans. Both observations $[1,2]$ and numerical simulations [3-5] have revealed the widespread appearance and dominance of vortices in turbulent geophysical flows. The prolific nature of vortices has led to their study becoming a field in its own right as a means to better understand and resolve the great unsolved problem of classical physics-turbulence.

While vorticity has a clear mathematical definition, being defined as a vector field which is the curl of the velocity flow field $(\boldsymbol{\omega}=\boldsymbol{\nabla} \times \mathbf{u})$, the definition of a vortex itself is far more elusive. This is due to the fact that a vortex does not have a particular shape. Ideas of tornadoes and hurricanes usually conjure up an image of circular or cylindrical type shapes for the vortex, but real vortices are not constrained to these perfect forms. However, when approaching them mathematically it is useful to apply a shape that is amenable to simple mathematical definition. One of the earliest incarnations of such an approach was that of Kirchhoff [6], who studied an isolated two-dimensional patch of uniform vorticity bounded within an ellipse. He showed that this is an exact analytical solution to the Euler equations which rotates with a constant angular velocity. Further work by Love [7] showed that there existed stable equilibria when this vortex is subjected to disturbances.

The extension of these early works to the threedimensional case of an ellipsoidal vortex had to wait almost a century for the works of Zhmur and Pankratov [8,9] and Meacham [10] who extended this approach to the quasigeostrophic system [11], a set of equations relevant to largescale geophysical fluid dynamics. In fact, the ellipsoidal form has a long history stretching back (at least) to the seminal works of MacClaurin [12] and Laplace [13] on gravitating bodies (see $[14,15]$ for a review of this subject). As the ellipsoidal form has an exact and simple mathematical definition 
that yet goes beyond the absolute symmetry of the sphere, it is a powerful tool in determining shape, structure, and orientation of "bodies" concerned in many different fields of application (see [16] for a review of the many applications). In the case of geophysical flows, it gives a means to determine the characteristics of vortices (such as typical height-to-width ratios, orientations, and rotation rates) that are the most robust and hence the most likely to occur in real turbulent flows.

Here we will review the recent history of the development of the ellipsoidal vortex in quasi-geostrophic dynamics. The beginning is in Section 2 where we give a brief overview of the governing system of equations, the quasi-geostrophic model [11]. Following this we review the ellipsoidal vortex model, starting in Section 3 with the case of the isolated ellipsoid as originally derived by Meacham [10]. In Section 4 we consider the case of an ellipsoidal vortex immersed in a background shear flow, following the matrix formulation approach of McKiver and Dritschel [17]. In Section 5 we discuss the results of studies of the equilibria and stability properties of this vortex system and what insights they provide for the full solution to the equations in turbulence simulations. In Section 6 we discuss the generalization of the single vortex ellipsoidal theory to the multiple vortex case, in particular outlining the Hamiltonian method of Dritschel et al. [18]. Finally we conclude in Section 7, with an overview of the consequences the ellipsoidal model has for the theory of turbulence and discuss future avenues of research in this field.

\section{The Quasi-Geostrophic System}

Here we summarize the quasi-geostrophic model (for a detailed derivation see [19]). Quasi-geostrophic (hereafter QG) theory is one of the simplest geophysical fluid dynamical models that incorporates two crucial features underpinning large-scale fluid dynamics: (1) the effect of the Earth's rotation and (2) the effects of strong density stratification (lighter fluid lying on top of denser fluid). As a consequence of these features certain terms in the full equations of motion (Navier-Stokes equations) tend to dominate over others. Strong rotation leads to what is termed "geostrophic balance," which is a balance between the horizontal pressure gradient and the term associated with the Earth's rotation (the Coriolis term):

$$
f \widehat{\mathbf{k}} \times \mathbf{u}_{h}=-\frac{\nabla p}{\rho_{0}},
$$

where $f$ is the local vertical component of the planetary rotation rate, $\widehat{\mathbf{k}}$ is a vertical unit vector, $\mathbf{u}_{h}$ is the horizontal velocity field, $p$ is pressure, and $\rho_{0}(z)$ is the basic-state density distribution ( $\rho_{0}$ is nearly constant in the oceans and exponential, proportional to $\exp (-z / H)$ with $H \approx 7 \mathrm{~km}$, in the atmosphere). Strong stratification leads to "hydrostatic balance," which is a balance between the vertical pressure gradient and buoyancy:

$$
\frac{\partial p}{\partial z}=-\rho_{0} g
$$

where $g$ is the acceleration due to gravity. The QG system, which incorporates both hydrostatic and geostrophic balance at leading order, is obtained from the full "primitive" equations by an asymptotic expansion in three small nondimensional parameters: (1) the height-width aspect ratio of characteristic motions $H / L$ (constrained to be small due to the shallow geometry in the oceans and atmosphere); (2) the Rossby number $R_{o} \equiv U / f L$; and (3) the Froude number $F_{r} \equiv U / N H$, where $U$ is a characteristic relative horizontal fluid speed, $L$ is the horizontal scale of the flow, $H$ is the average fluid depth, and $N$ is the buoyancy frequency (the oscillation frequency that a small fluid volume would exhibit if it were displaced by a small vertical distance). The QG model is obtained assuming that $H / L \ll 1$ and $F_{r}^{2} \ll R_{o} \ll 1$. Also in this model we consider the limit of infinite Reynolds number, $\operatorname{Re} \equiv U L / v$; that is, the effect of viscous dissipation, $\nu$, is negligible (in the real atmosphere, $\operatorname{Re} \sim 10^{10}-10^{12}$ ). As done in many previous works, one can consider the constantcoefficient ("oceanic") system when $f, N$, and $\rho_{0}$ are all constant. For an inviscid, adiabatic fluid, the QG equations may be expressed in terms of the conservation of a single material scalar, the "potential vorticity," $q(\mathbf{x}, t)$; that is,

$$
\frac{D q}{D t} \equiv \frac{\partial q}{\partial t}+u \frac{\partial q}{\partial x}+v \frac{\partial q}{\partial y}=0
$$

The potential vorticity (hereafter PV) is proportional to the component of vorticity perpendicular to stratification surfaces (surfaces of constant density $\rho_{0}$ ). Thus vortices are identified as contiguous regions of PV. In the QG model, the vertical velocity $w=0$ to this order of approximation, so $q$ is advected in a layerwise manner. However the horizontal velocity $(u, v)$ depends on all three spatial coordinates, and this is recovered from $q$ via the inversion of

$$
\nabla^{2} \psi=q
$$

for the stream function $\psi$, followed by the incompressibility relations

$$
u=-\frac{\partial \psi}{\partial y}, \quad v=\frac{\partial \psi}{\partial x} .
$$

Note that in this form the vertical coordinate $z$ is rescaled by the factor $f / N$. The three-dimensional distribution of PV generates the velocity field through (4) and (5), which carries the PV, conservatively, to the next instant of time. While (4) is an isotropic relation between $q$ and $\psi$, the advection remains anisotropic as there is no vertical motion. The QG equations are widely used in studies of atmospheric and oceanic phenomena [20-23], in part because they are far easier to deal with both theoretically and numerically than the complete equations. But, also, the QG equations capture many qualitative features of atmospheric and oceanic dynamics.

\section{Potential Theory of an Isolated Ellipsoidal Vortex}

Now we consider the case of an isolated ellipsoidal vortex, with uniform potential vorticity, as first considered in the 
works of Zhmur and Pankratov [8,9] (see also [24]) and independently by Meacham [10]. We will review the full solution to the QG ellipsoid as derived by Meacham [10]. We consider the ellipsoid of PV having semiaxes lengths $a, b$, and $c$, where $a \geq b \geq c$. Without loss of generality we assume the vortex axes are aligned along the coordinate axis. Thus the boundary of the ellipsoid is defined by the equation

$$
\frac{x^{2}}{a^{2}}+\frac{y^{2}}{b^{2}}+\frac{z^{2}}{c^{2}}=1
$$

Inside the ellipsoid we have from (4)

$$
\nabla^{2} \psi_{i}=q
$$

where $q$ is the uniform potential vorticity. A general solution to this equation can be written as

$$
\psi_{i}=\frac{1}{2}\left(f x^{2}+g y^{2}+h z^{2}\right)+C_{i}
$$

where $f, g, h$, and $C_{i}$ are unknown coefficients to be determined. Substituting this into (7) gives the condition

$$
f+g+h=q \text {. }
$$

Outside the ellipsoid the potential vorticity is zero and the external potential satisfies

$$
\nabla^{2} \psi_{e}=0
$$

With such a problem the natural approach is to move from Cartesian coordinates $(x, y, z)$ to an ellipsoidal coordinate system $(\lambda, \mu, \nu)$ using the relations [25]

$$
\begin{aligned}
& x^{2}=\frac{\left(\lambda+a^{2}\right)\left(\mu+a^{2}\right)\left(\nu+a^{2}\right)}{\left(a^{2}-b^{2}\right)\left(a^{2}-c^{2}\right)}, \\
& y^{2}=\frac{\left(\lambda+b^{2}\right)\left(\mu+b^{2}\right)\left(\nu+b^{2}\right)}{\left(b^{2}-c^{2}\right)\left(b^{2}-a^{2}\right)}, \\
& z^{2}=\frac{\left(\lambda+c^{2}\right)\left(\mu+c^{2}\right)\left(\nu+c^{2}\right)}{\left(c^{2}-a^{2}\right)\left(c^{2}-b^{2}\right)},
\end{aligned}
$$

where the variables $\lambda, \mu$, and $\nu$ represent the roots to the cubic equation

$$
\frac{x^{2}}{a^{2}+\chi}+\frac{y^{2}}{b^{2}+\chi}+\frac{z^{2}}{c^{2}+\chi}=1,
$$

where $\lambda>-c^{2}>\mu>-b^{2}>v>-a^{2}$. The $\lambda$-surfaces are ellipsoids, the $\mu$-surfaces are hyperboloids of one sheet, and the $\nu$-surfaces are hyperboloids of two sheets [16]. The largest root, $\lambda$, is analogous to the radial variable in spherical coordinates with each $\lambda$ representing a family of concentric ellipsoids, with $\lambda=0$ being the value on the surface of the original ellipsoid with semiaxes $a, b$, and $c$. The boundary conditions on the surface of the ellipsoid are the continuity of the normal and tangential velocity components, which can be expressed in ellipsoidal coordinates as

$$
\begin{aligned}
\left.\psi_{i}\right|_{\lambda=0} & =\left.\psi_{e}\right|_{\lambda=0}, \\
\left.\frac{\partial \psi_{i}}{\partial \lambda}\right|_{\lambda=0} & =\left.\frac{\partial \psi_{e}}{\partial \lambda}\right|_{\lambda=0} .
\end{aligned}
$$

Substituting (11a), (11b), and (11c) into the expression for the internal field (8) we obtain

$$
\begin{aligned}
\psi_{i}= & \frac{f\left(\lambda+a^{2}\right)\left[\mu \nu+a^{2}(\mu+\nu)+a^{4}\right]}{2\left(a^{2}-b^{2}\right)\left(a^{2}-c^{2}\right)} \\
& +\frac{g\left(\lambda+b^{2}\right)\left[\mu \nu+b^{2}(\mu+\nu)+b^{4}\right]}{2\left(b^{2}-c^{2}\right)\left(b^{2}-a^{2}\right)} \\
& +\frac{h\left(\lambda+c^{2}\right)\left[\mu \nu+c^{2}(\mu+\nu)+c^{4}\right]}{2\left(c^{2}-a^{2}\right)\left(c^{2}-b^{2}\right)}+C_{i} .
\end{aligned}
$$

A general solution for the external flow field can be written as an expansion in ellipsoidal harmonics $[16,25,26]$

$$
\phi_{e}=\sum_{m}^{\infty} \sum_{k}^{2 m+1} \mathbf{F}_{m}^{(k)}(\lambda) \mathbf{E}_{m}^{(k)}(\mu) \mathbf{E}_{m}^{(k)}(\nu),
$$

where $\mathbf{E}_{m}^{(k)}$ denote Lame functions of the first kind, while $\mathbf{F}_{m}^{(k)}$ are Lame functions of the second kind defined by

$\mathbf{F}_{m}^{(k)}(\lambda)$

$$
\begin{aligned}
= & \frac{2 m+1}{2} \mathbf{E}_{m}^{(k)}(\lambda) \\
& \cdot \int_{0}^{\infty} \frac{d s}{\left[\mathbf{E}_{m}^{(k)}(s+\lambda)\right]^{2} \sqrt{\left(s+\lambda+a^{2}\right)\left(s+\lambda+b^{2}\right)\left(s+\lambda+c^{2}\right)}} .
\end{aligned}
$$

The dependence on the coordinates $\mu$ and $\nu$ for the inner field in (14) implies that the exterior solution needed in order to match the interior solution at the boundary has the form

$$
\begin{aligned}
\phi_{e}= & C_{0} \mathbf{F}_{0}^{(1)}(\lambda)+C_{2}^{(1)} \mathbf{F}_{2}^{(1)}(\lambda) \mathbf{E}_{2}^{(1)}(\mu) \mathbf{E}_{2}^{(1)}(\nu) \\
& +C_{2}^{(2)} \mathbf{F}_{2}^{(2)}(\lambda) \mathbf{E}_{2}^{(2)}(\mu) \mathbf{E}_{2}^{(2)}(\nu),
\end{aligned}
$$

where $C_{0}, C_{2}^{(1)}$, and $C_{2}^{(2)}$ are unknown coefficients and the second-order Lame functions of the first kind are

$$
\begin{aligned}
& \mathbf{E}_{2}^{(1)}(\lambda)=\lambda+d_{1}, \\
& \mathbf{E}_{2}^{(2)}(\lambda)=\lambda+d_{2},
\end{aligned}
$$

where

$$
\begin{aligned}
d_{1}=\frac{1}{3}[ & \left(a^{2}+b^{2}+c^{2}\right) \\
& \left.+\sqrt{a^{4}+b^{4}+c^{4}-b^{2} c^{2}-c^{2} a^{2}-a^{2} b^{2}}\right], \\
d_{2}=\frac{1}{3}[ & \left(a^{2}+b^{2}+c^{2}\right) \\
& \left.-\sqrt{a^{4}+b^{4}+c^{4}-b^{2} c^{2}-c^{2} a^{2}-a^{2} b^{2}}\right] .
\end{aligned}
$$


Substituting these Lame functions into the external field gives us

$$
\begin{aligned}
\psi_{e}= & \mu \nu\left[C_{2}^{(1)} \mathbf{F}_{2}^{(1)}(\lambda)+C_{2}^{(2)} \mathbf{F}_{2}^{(2)}(\lambda)\right] \\
& +(\mu+\nu)\left[d_{1} C_{2}^{(1)} \mathbf{F}_{2}^{(1)}(\lambda)+d_{2} C_{2}^{(2)} \mathbf{F}_{2}^{(2)}(\lambda)\right] \\
& +C_{0} \mathbf{F}_{0}^{(1)}(\lambda)+d_{1}^{2} C_{2}^{(1)} \mathbf{F}_{2}^{(1)}(\lambda)+d_{2}^{2} C_{2}^{(2)} \mathbf{F}_{2}^{(2)}(\lambda) .
\end{aligned}
$$

Substituting (14) and (20) into the boundary conditions equations (13a) and (13b) provides 6 conditions, since there are terms proportional to $\mu \nu$ and $\mu+\nu$ as well as the constant terms. These 6 conditions along with the condition given by (9) provide 7 equations in the 7 unknown coefficients and so can be solved as was shown by Meacham [10]. Solving these simultaneous equations we can write the interior stream function as

$$
\psi_{i}=\frac{1}{2}\left(\xi_{a} x^{2}+\xi_{b} y^{2}+\xi_{c} z^{2}\right)-\frac{3}{2} \kappa_{v} R_{F}\left(a^{2}, b^{2}, c^{2}\right),
$$

where $\xi_{a}, \xi_{b}$, and $\xi_{c}$ are constant coefficients given by

$$
\begin{aligned}
& \xi_{a}=\kappa_{v} R_{D}\left(b^{2}, c^{2}, a^{2}\right), \\
& \xi_{b}=\kappa_{v} R_{D}\left(c^{2}, a^{2}, b^{2}\right), \\
& \xi_{c}=\kappa_{v} R_{D}\left(a^{2}, b^{2}, c^{2}\right),
\end{aligned}
$$

where $\kappa_{v}=q a b c / 3$ is the vortex "strength" and $R_{F}$ and $R_{D}$ are elliptic integrals of the first and second kind, respectively, defined as

$$
\begin{gathered}
R_{F}(x, y, z) \equiv \frac{1}{2} \int_{0}^{\infty} \frac{d t}{\sqrt{(t+x)(t+y)(t+z)}}, \\
R_{D}(x, y, z) \equiv \frac{3}{2} \int_{0}^{\infty} \frac{d t}{\sqrt{(t+x)(t+y)(t+z)^{3}}} .
\end{gathered}
$$

This solution was originally determined by Laplace [13] for the case of a gravitating ellipsoid of uniform mass [14]. The exterior stream function is

$$
\psi_{e}=\frac{1}{2}\left(\xi_{\alpha} x^{2}+\xi_{\beta} y^{2}+\xi_{\gamma} z^{2}\right)-\frac{3}{2} \kappa_{v} R_{F}\left(\alpha^{2}, \beta^{2}, \gamma^{2}\right),
$$

where $\alpha^{2}=a^{2}+\lambda, \beta^{2}=b^{2}+\lambda$, and $\gamma^{2}=c^{2}+\lambda$ and now we have

$$
\begin{aligned}
& \xi_{\alpha}=\kappa_{v} R_{D}\left(\beta^{2}, \gamma^{2}, \alpha^{2}\right), \\
& \xi_{\beta}=\kappa_{v} R_{D}\left(\gamma^{2}, \alpha^{2}, \beta^{2}\right), \\
& \xi_{\gamma}=\kappa_{v} R_{D}\left(\alpha^{2}, \beta^{2}, \gamma^{2}\right) .
\end{aligned}
$$

While the interior solution has quadratic dependence on spatial coordinates, the exterior field has a more complicated dependence, due to the coefficients being functions of the variable $\lambda$. However, the flow field that acts on the vortex itself is the interior solution, and so one can compute the flow field acting on the vortex itself from (5). This gives rise to a selfinduced velocity field that is linear in spatial coordinates.

\section{Evolution of a Vortex in a Background Shear Flow}

We now consider the case of an ellipsoidal vortex immersed in a background shear flow. This background shear flow idealizes the effects of other vortices and so is the first step towards understanding interactions between vortices. This case was first considered by Meacham et al. [27] for a number of specific types of background flow and later generalized by Meacham et al. [28] where it was recast within a Hamiltonian framework. Hashimoto et al. [29] extended the analytical solutions of Meacham [10] for the isolated vortex by solving for exact equilibrium solutions for the case of the ellipsoid in an external straining flow. McKiver and Dritschel [17] introduced a reformulation of the system that greatly simplified the equations to a simple $3 \times 3$ matrix problem. In this section we review their derivation of this matrix formulation. This approach is built on the simple fact that the stream function for the ellipsoid has quadratic dependence on spatial coordinates, and so the velocity field has a linear dependence. Naturally any linear flow field imposed on the ellipsoidal vortex, while possibly altering the size $(a, b$, and $c)$ and orientation of the vortex, preserves the ellipsoidal form. Thus we consider a general velocity field of the form

$$
\mathbf{u}_{b}(\mathbf{x}, t)=\mathbf{S}_{b}(t) \mathbf{x}
$$

where $\mathbf{S}_{b}$ is a $3 \times 3$ matrix. Within this flow, we place a single ellipsoidal vortex at the origin and require only that its velocity field has the same form as (26); that is, $\mathbf{u}_{v}=\mathbf{S}_{v} \mathbf{x}$. The total velocity field felt by the ellipsoid is

$$
\mathbf{u}=\mathbf{S} \mathbf{x},
$$

where $\mathbf{S}=\mathbf{S}_{v}+\mathbf{S}_{b}$, and this depends only on time $t$. We refer to $\mathbf{S}$ as the flow matrix subsequently.

The ellipsoid is specified by its axis half lengths $a \geq b \geq$ $c$ and the unit vectors $\widehat{\mathbf{a}}, \widehat{\mathbf{b}}$, and $\widehat{\mathbf{c}}$ directed along these axes. This geometric information may be encapsulated in a single symmetric matrix A defined by

$$
\mathbf{A}=\mathbf{M D M}^{T}
$$

where the superscript $T$ denotes transpose, $\mathbf{M}$ is an orthonormal rotation matrix $\left(\mathbf{M}^{-1}=\mathbf{M}^{T}\right)$ defined by

$$
\mathbf{M}=\left(\begin{array}{lll}
\widehat{\mathbf{a}} & \widehat{\mathbf{b}} & \widehat{\mathbf{c}}
\end{array}\right)
$$

and $\mathbf{D}$ is a diagonal matrix whose terms are the inverse squared semiaxes lengths:

$$
\mathbf{D}=\left(\begin{array}{ccc}
\frac{1}{a^{2}} & 0 & 0 \\
0 & \frac{1}{b^{2}} & 0 \\
0 & 0 & \frac{1}{c^{2}}
\end{array}\right)
$$

The equation describing the surface of the ellipsoid is conveniently written as

$$
\mathbf{x}^{T} \mathbf{A x}=1 .
$$


By taking a time derivative of this equation and using $d \mathbf{x} / d t=$ $\mathbf{u}(\mathbf{x}, t)=\mathbf{S}(t) \mathbf{x}$, as well as the implied relation $d \mathbf{x}^{T} / d t=$ $\mathbf{x}^{T} \mathbf{S}^{T}(t)$, we obtain

$$
\mathbf{x}^{T}\left(\mathbf{A S}+\mathbf{S}^{T} \mathbf{A}+\frac{d \mathbf{A}}{d t}\right) \mathbf{x}=0
$$

Since this must be true for all points $\mathbf{x}$ on the boundary of the ellipsoid, it follows that

$$
\frac{d \mathbf{A}}{d t}=-\left(\mathbf{A S}+\mathbf{S}^{T} \mathbf{A}\right)
$$

which is simply the equation for the evolution of $\mathbf{A}$ in an arbitrary linear background flow. Note that $\mathbf{A}$ remains a symmetric matrix for all time since $\mathbf{A S}+\mathbf{S}^{T} \mathbf{A}$ is automatically symmetric if $\mathbf{A}$ is.

It proves convenient to rewrite (33) to evolve not $\mathbf{A}$ but its inverse $\mathbf{B}=\mathbf{A}^{-1}$. Using the fact that the product $\mathbf{A B}$ is the identity matrix, it follows that

$$
\begin{aligned}
\frac{d \mathbf{B}}{d t} & =-\mathbf{B} \frac{d \mathbf{A}}{d t} \mathbf{B} \\
& =\mathbf{B}\left(\mathbf{A S}+\mathbf{S}^{T} \mathbf{A}\right) \mathbf{B}
\end{aligned}
$$

giving us the evolution equation (as originally derived in [17])

$$
\frac{d \mathbf{B}}{d t}=\mathbf{S B}+\mathbf{B S}^{T}
$$

where

$$
\begin{gathered}
\mathbf{B}=\mathbf{M D}^{-1} \mathbf{M}^{T}, \\
\mathbf{D}^{-1}=\left(\begin{array}{ccc}
a^{2} & 0 & 0 \\
0 & b^{2} & 0 \\
0 & 0 & c^{2}
\end{array}\right) .
\end{gathered}
$$

In order to determine the evolution of the ellipsoid it is necessary to be able to calculate the semiaxes lengths, $a, b$, and $c$, and the orientation vectors $\widehat{\mathbf{a}}, \widehat{\mathbf{b}}$, and $\widehat{\mathbf{c}}$ of the ellipsoid from the matrix B. But (36), right multiplied by $\mathbf{M}$, together with (37) implies

$$
\begin{aligned}
& \mathbf{B} \widehat{\mathbf{a}}=a^{2} \widehat{\mathbf{a}}, \\
& \mathbf{B} \widehat{\mathbf{b}}=b^{2} \widehat{\mathbf{b}}, \\
& \mathbf{B} \widehat{\mathbf{c}}=c^{2} \widehat{\mathbf{c}}
\end{aligned}
$$

Hence the semiaxes lengths and the orientation vectors of the ellipsoid can be found directly from the $\mathbf{B}$ matrix by solving a simple eigenvalue problem. Finally one must specify the flow matrix $\mathbf{S}=\mathbf{S}_{v}+\mathbf{S}_{b}$. The self-induced part is derived from the stream function solution to the inner potential problem, $\psi_{v}$. This solution can be written more generally for an arbitrarily orientated ellipsoid as

$$
\psi_{v}=\frac{1}{2} \mathbf{x}^{T} \mathbf{P}_{v} \mathbf{x}-\frac{3}{2} \kappa_{v} R_{F}\left(a^{2}, b^{2}, c^{2}\right)
$$

where

$$
\mathbf{P}_{v}=\mathbf{M G M}^{T}
$$

and $\mathbf{G}$ is the diagonal matrix formed from the solution coefficients:

$$
\mathbf{G}=\left(\begin{array}{ccc}
\xi_{a} & 0 & 0 \\
0 & \xi_{b} & 0 \\
0 & 0 & \xi_{c}
\end{array}\right)
$$

From (5) it follows that

$$
\mathbf{S}_{v}=\mathbf{L P}_{v}
$$

where $\mathbf{L}$ is defined as

$$
\mathbf{L}=\left(\begin{array}{ccc}
0 & -1 & 0 \\
1 & 0 & 0 \\
0 & 0 & 0
\end{array}\right)
$$

For the background flow matrix one can consider the effect of a single distant vortex, of strength $\kappa_{b}$, centered at $\mathbf{x}=\mathbf{X}_{b}$. Let $R=\left|\mathbf{X}_{b}-\mathbf{X}_{v}\right|$ be the distance between the two vortices; then, to leading order in $1 / R$ the vortices rotate about each other at a rate

$$
\Omega=\frac{\kappa_{b}+\kappa_{v}}{R^{3}}
$$

At this order of approximation the vortices appear as points, with no shape or internal structure. If we adopt a frame of reference rotating with the $z$-axis passing through the joint centre of the two vortices, so that their positions $\mathbf{X}_{v}$ and $\mathbf{X}_{b}$ are fixed, and assuming the original vortex is located at the origin, $\mathbf{X}_{v}=0$, then in the vicinity of the origin the background stream function takes the form (Taylor expanding)

$$
\psi_{b}=-\frac{\kappa_{b}}{R}+\frac{1}{2} \mathbf{x}_{T} \mathbf{P}_{b} \mathbf{x}+O\left(\frac{\kappa_{b}|\mathbf{x}|^{3}}{R^{4}}\right),
$$

where notably the linear terms are absent and the matrix $\mathbf{P}_{b}$ is given by

$$
\begin{aligned}
\mathbf{P}_{b}= & \frac{\kappa_{b}}{R^{5}}\left(\begin{array}{ccc}
R^{2}-3 X_{b}^{2} & -3 X_{b} Y_{b} & -3 X_{b} Z_{b} \\
-3 X_{b} Y_{b} & R^{2}-3 Y_{b}^{2} & -3 Y_{b} Z_{b} \\
-3 X_{b} Z_{b} & -3 Y_{b} Z_{b} & R^{2}-3 Z_{b}^{2}
\end{array}\right) \\
& -\left(\begin{array}{ccc}
\Omega & 0 & 0 \\
0 & \Omega & 0 \\
0 & 0 & 0
\end{array}\right) .
\end{aligned}
$$

The corresponding velocity field is given by $\mathbf{u}_{b}=\mathbf{S}_{b} \mathbf{x}$, where as before the flow matrix is $\mathbf{S}_{b}=\mathbf{L} \mathbf{P}_{b}$, leading to

$$
\mathbf{S}_{b}=\gamma\left(\begin{array}{ccc}
3 \widetilde{X} \tilde{Y} & 3 \widetilde{Y}^{2}-1+\beta & 3 \widetilde{Y} \widetilde{Z} \\
1-\beta-3 \widetilde{X}^{2} & -3 \widetilde{X} \tilde{Y} & -3 \widetilde{X} \widetilde{Z} \\
0 & 0 & 0
\end{array}\right)
$$

where $\widetilde{\mathbf{X}} \equiv \mathbf{X}_{b} / R$,

$$
\gamma \equiv \frac{\kappa_{b}}{R^{3}}
$$


hereinafter referred to as "the strain rate," and

$$
\beta \equiv \frac{\Omega}{\gamma}=\frac{\kappa_{b}+\kappa_{v}}{\kappa_{b}}
$$

is a parameter depending only on the ratio of the vortex strengths. It can be simplified further by choosing either $\widetilde{X}$ or $\widetilde{Y}$ to be zero, leaving only three nonzero components. For example, if $\widetilde{X}=0$,

$$
\mathbf{S}_{b}=\gamma\left(\begin{array}{ccc}
0 & \frac{1}{2}(1+3 \cos 2 \theta)+\beta & \frac{3}{2} \sin 2 \theta \\
1-\beta & 0 & 0 \\
0 & 0 & 0
\end{array}\right)
$$

where $\widetilde{Z}=\sin \theta, \widetilde{Y}=\cos \theta$, and the range of the angle $\theta$ is restricted to $\left[0^{\circ}, 90^{\circ}\right]$ because of symmetry. This form for the background flow matrix was originally considered by Meacham et al. [27] but using a different notation.

The complete model now consists of the evolution equation (35) for the matrix $\mathbf{B}$ together with expressions (42) and (50) for the flow matrices $\mathbf{S}_{v}$ and $\mathbf{S}_{b}$. Note that $d \mathbf{B}_{33} / d t=0$ in QG flow-this follows because there is no vertical velocity. The element $\mathbf{B}_{33}$ is the squared half-height of the vortex. Note also that the determinant $|\mathbf{B}|=\left|\mathbf{D}^{-1}\right|=(a b c)^{2}$ is proportional to the squared vortex volume and is also invariant (because QG flow is incompressible).

\section{Equilibria and Stability}

Given the equations governing the evolution of an ellipsoidal vortex in a background shear flow one can then determine equilibrium vortices from (35), where they satisfy

$$
\mathbf{S B}+\mathbf{B S}^{T}=0 .
$$

This was first exploited by Reinaud et al. [30] using an iterative linear method to determine equilibria. These equilibria have important consequences for vortex interactions. As a vortex evolves over its lifetime it may come into close proximity with other vortices. When this happens the strain exerted on one vortex by the others increases, as the magnitude of this strain scales with the inverse cube of the separation distance between vortices (see (48)). At some "critical" value of strain the vortex will begin to deform significantly and will undergo a strong interaction. If the vortices are close enough they may partially or fully coalesce or "merge" into one, forming a larger vortex along with filamentary debris. Often the onset of such strong interactions occurs at the point beyond which equilibria can no longer exist for strains greater than this critical value, or beyond which equilibria are unstable to disturbances. Thus the stability analysis of a vortex in equilibrium with a background shear is an important indicator of when strong interactions may occur. Next we give a brief summary of the findings of a number of studies on this topic.

The stability of a freely rotating ellipsoid of potential vorticity was first considered by Meacham [10] who found instabilities over a large range of the parameter space characterising the ellipsoid. However, a more recent study by
Dritschel et al. [31] found that one of the unstable modes Meacham encountered is in fact stable, and overall their results indicate that the freely rotating ellipsoid is widely stable.

Miyazaki et al. [32] studied the stability of a spheroid of uniform PV tilted by some inclination angle from the vertical axis. They found that highly prolate spheroids are unstable if the inclination angle is large, while oblate spheroids are unstable even if the inclination angle is very small. In both cases the instability is nonellipsoidal; that is, it destroys the ellipsoidal form of the vortex.

Hashimoto et al. [29] derived the equations for the linear stability of an ellipsoid in a 2D strain field by expanding the disturbing surface in Lame functions. Using these they studied the cases of a pure strain field (when there is no background rotation) and a simple shear flow (when the strain rate is equal to the background rotation). They found that, in a pure strain field, highly elongated ellipsoids are unstable to modes whose order $m$ is greater than 2 , that is, to nonellipsoidal modes. In a simple shear flow they found that a highly elongated ellipsoid whose major axis is perpendicular to the flow direction is unstable, whereas any ellipsoidal vortex seems to be stable if the major axis is parallel to the flow direction.

Meacham et al. [27] derived equations for the evolution of an ellipsoid of uniform PV in a background flow with both horizontal strain and vertical shear. They examined the stability for some special background flows, either pure horizontal strain or pure vertical shear (in the absence of horizontal strain). A full linear stability analysis for the case of a general linear background flow (50) was conducted by McKiver and Dritschel [33]. They characterized the system by four parameters: the height-to-width aspect ratio of the vortex $h / r$, the strength ratio $\beta$, the angle $\theta$, and the strain rate $\gamma$. Over nearly the entire parameter space examined they found that the ellipsoidal steady states are stable, and where instability does occur the dominant modes encountered are ellipsoidal. Thus the ellipsoidal vortex in a linear background flow is a fairly robust model. They also found that for vortices with aspect ratios greater than 0.8 the most unstable vortices (i.e., the ones which destabilize at the smallest strain values $\gamma$ ) are found in the ranges $55^{\circ} \leq \theta \leq 75^{\circ}$ and $20^{\circ} \leq$ $\theta \leq 30^{\circ}$, for opposite- and like-signed vortices, respectively. Because the strain is proportional to the inverse cube of the separation distances between two vortices, this implies that vortices which are vertically offset by these $\theta$ values destabilize from the greatest separation distances; that is, they are least resilient. This agrees with the findings of Reinaud and Dritschel [34] in which they examined the merger of two identical uniform PV vortices which are offset both horizontally and vertically and found that vortices which are moderately offset in the vertical merge from a greater separation distance than vortices that are not offset vertically. In McKiver and Dritschel [33], for opposite-signed vortices, they found that the most stable vortices have an aspect ratio between 1.0 and 1.25, whereas for the like-signed vortices the most stable vortices have an aspect ratio near 0.8 . This agrees with the findings in a study by Reinaud et al. [30] where they performed simulations of a turbulent flow comprising 
hundreds of QG vortices in order to determine the typical shape of vortices in QG turbulence. Remarkably their study also found that typically vortices are slightly oblate having a height-to-width aspect ratio of $H / L \approx 0.8$.

\section{Multiple Ellipsoidal Vortices}

While the single vortex case provides great insight into vortex behaviour, in order to understand some of the dynamics that precipitate strong interactions and vortex mergers, one must consider a multivortex scenario. There are a number of works that have generalized the single ellipsoidal vortex solution to a multivortex scenario [35-38]. However, because of the form of the external stream function (24) generated by a vortex, in general it can induce nonellipsoidal disturbances on external vortices. This means that assumptions must be made in order to retain the ellipsoidal form. Here we will briefly summarize an accurate approach introduced by Dritschel et al. [18]. This approach, referred to as ELM, represents vortices as PV ellipsoids and filters higher-order nonellipsoidal deformations simply by preserving only the linear part of the exterior field. Following the matrix formulation in Section 4 each vortex can be specified by the value of its potential vorticity, $q_{i}$, the symmetric matrix $\mathbf{B}_{i}$, and the location of the center of the vortex $\mathbf{X}_{i}=\left(X_{i}, Y_{i}, Z_{i}\right)$, in terms of which the surface of each ellipsoid can be written as

$$
\left(\mathbf{x}-\mathbf{X}_{i}\right)^{T} \mathbf{B}_{i}^{-1}\left(\mathbf{x}-\mathbf{X}_{i}\right)=1,
$$

where $i=1, \ldots, N$, where $N$ is the number of vortices. If we consider that the velocity field for a particular ellipsoid $\left(q_{i}, \mathbf{X}_{i}, \mathbf{B}_{i}\right)$ has a linear dependence on spatial coordinates, that is,

$$
\mathbf{u}_{i}(\mathbf{x}, t)=\mathbf{U}_{i}(t)+\mathbf{S}_{i}(t)\left(\mathbf{x}-\mathbf{X}_{i}\right),
$$

where $\mathbf{U}_{i}(t)$ is an arbitrary velocity field and $\mathbf{S}_{i}(t)$ is the flow matrix, then this velocity field is the most general form which exactly preserves the ellipsoidal form of the vortex. The governing equation for ELM [18] is a finite Hamiltonian system where the equations of motion for the $i$ th ellipsoid are

$$
\begin{aligned}
\frac{d \mathbf{X}_{i}}{d t} & =-\frac{1}{\kappa_{i}} \mathbf{L} \frac{\partial H}{\partial \mathbf{X}_{i}}, \\
\frac{d \mathbf{B}_{i}}{d t} & =\mathbf{S}_{i} \mathbf{B}_{i}+\mathbf{B}_{i} \mathbf{S}_{i}^{T}, \\
\mathbf{S}_{i} & =-\frac{10}{\kappa_{i}} \mathbf{L} \frac{\partial H}{\partial \mathbf{B}_{i}},
\end{aligned}
$$

where $\kappa_{i}$ is the vortex strength, $H$ is the Hamiltonian of the system defined as the total energy of the system divided by $4 \pi$, and the flow matrix is decomposed into two parts, $\mathbf{S}_{i}=\mathbf{S}_{i}^{v}+\mathbf{S}_{i}^{b}$, the self-induced part $\mathbf{S}_{i}^{v}$ and the part induced by all other ellipsoids $S_{i}^{b}$. For efficiency of the method this background flow field is modelled for each ellipsoid as a finite sum of singular point vortices. The position and strength of the singularities are obtained such that the approximate stream function induced by them matches
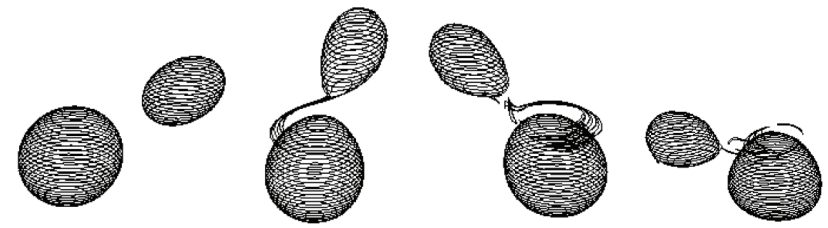

(a)
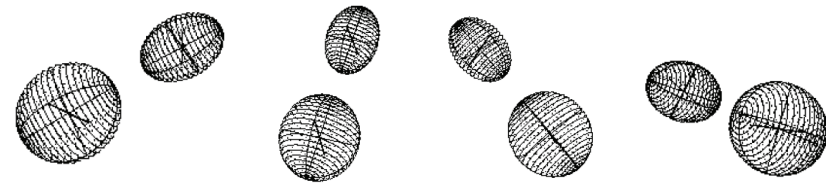

(b)

FIgURE 1: The time evolution of two like-signed vortices solved using contour dynamics solution of the full QG equations (a) and using the ellipsoidal model (b). The side view is from an angle of 60 degrees. Figures courtesy of http://www-vortex.mcs.st-and.ac.uk/ $\operatorname{dgd} / \mathrm{ELM} /$.

exactly the function at a given order of accuracy in $1 / R$, $R$ being the distance from $\mathbf{X}_{i}$ to the point of evaluation. Dritschel et al. [18] find that high accuracy $\left(O\left(1 / R^{7}\right)\right)$ can typically be achieved by using 7 point singularities for each ellipsoid. Comparisons of the ELM with full numerical simulations (Figure 1) of the QG vortices show remarkable agreement (see http://www-vortex.mcs.st-and.ac.uk/ dgd/ $\mathrm{ELM} /$ to see more comparisons).

The steady states for the case of a two-vortex ELM system were first analyzed by Reinaud and Dritschel [39]. In this case, the parameters considered were the volume ratios $\left(\rho_{V}=\right.$ $\left.V_{2} / V_{1}\right)$, the vertical offsets $(\Delta z)$, and height-to-width aspect ratios $(h / r)$. As with the single vortex case, the method to determine equilibria involves the solution of

$$
\mathbf{S}_{i} \mathbf{B}_{i}+\mathbf{B}_{i} \mathbf{S}_{i}^{T}=0
$$

in the rotating reference frame where $d \mathbf{X}_{i} / d t=0$. A family of equilibria for a given set of parameters $\left(\rho_{V}, \Delta z, h / r\right)$ are found for different horizontal distances between the vortices, $\delta$, where $\delta$ is defined as the horizontal gap between the two innermost edges of the ellipsoids; specifically

$$
\delta=\left|\mathbf{X}_{1}-\mathbf{X}_{2}\right|-\sqrt{\left(\mathbf{B}_{11}\right)_{1}}-\sqrt{\left(\mathbf{B}_{11}\right)_{2}} .
$$

Below a critical value of $\delta$ the equilibria become unstable inducing strong interactions that may lead to merger of the two vortices. Analyzing the stability of these equilibria Reinaud and Dritschel [39] discovered a "tilt" instability that results in the merger of prolate vortices from greater separation distances than seen in previous symmetric merger cases (i.e., no vertical offset). This tilt instability is the first instability able to precipitate the merger of prolate vortices. The merger of corotating vortices was also studied in detail by Li et al. [37] where they introduced a new set of nearly canonical variables to solve the multivortex system. The case of two counterrotating ellipsoidal vortices having the same size was considered by Miyazaki et al. [40]. They found that 
in general the vortex pair propagate as a stable dipole, except for certain tall vortices; when initially placed within a critical distance, they begin to tilt and eventually destabilize.

\section{Conclusions}

Here we reviewed the recent history of the theory of ellipsoidal vortices in the field of geophysical fluid dynamics. The strength and utility of this model is that it provides analytical vortex solutions whose equilibria and stability can easily be determined. Remarkably this simple model can provide insights into the dynamics seen in the full solutions of the governing equations in complicated turbulence simulations. This is clearly seen even for the single vortex in a background shear flow where the most stable aspect ratio of $\sim 0.8[33]$ is the ratio statistically most seen in complex QG turbulence simulations [30].

An interesting recent study by Koshel et al. [41] modified the ellipsoidal model, adding diffusion, in order to study how passive scalar transport through the vortex boundary is affected by both advection and diffusion. They showed that adding disturbances to the vortex can boost the rate of transport of the tracer out of the vortex relative to the undisturbed case and discuss the potential of this model as a means to determine diffusivity values in the real ocean. This study is one example of the potential applications that the ellipsoidal model can have for studying processes in the ocean and atmosphere, where it has the advantage of an analytical solution for the flow field.

The application of the ellipsoidal vortex model discussed in this review has only been for the quasi-geostrophic equations, which applies to large-scale dynamics when the Rossby number is small. However the QG equations are only the first order in a hierarchy of the so-called "balanced" models (i.e., based on geostrophic and hydrostatic balance) that capture the underlining effects of rotation and stratification. A very recent numerical study by Tsang and Dritschel [42] looked at the evolution of an ellipsoidal vortex for more general dynamics beyond the QG limit. They found that these ellipsoidal vortices again are very robust structures, being to a large extent quasi-equilibria over long time integrations. This prompted McKiver and Dritschel [43] to revisit the potential problem for an isolated ellipsoid using equations at the next order to the QG theory (so-called QG+1). In this higher-order model, one encounters three Poisson equations as the velocity field is now defined in terms of a vector potential instead of a scalar stream function as in the QG case. They showed that an analytical solution can be found to this QG+1 system of equations analogous to the QG case. This is a powerful extension of the ellipsoidal theory in geophysical fluid dynamics having the potential to capture many features not seen in the $\mathrm{QG}$ limit. For one thing in the QG+1 model there is a vertical velocity unlike the QG model. Another limitation of the QG model is that there is complete symmetry between cyclonic and anticyclonic vortices, whereas in reality the Earth rotates in one direction giving rise to an asymmetry in the behaviour of cyclones and anticyclones. The next order to QG picks up this asymmetry and so is much more applicable to a wider range of geophysical flows. Potential future studies of these higher-order solutions could determine equilibria and their stability properties providing insights into turbulence beyond the large-scale approximation. Also the development of a multiellipsoidal vortex theory beyond QG would have huge benefits in understanding more realistic turbulence phenomena. The exterior field in QG+1 is much more complicated than the QG case and so the projection of this onto a linear flow field that preserves ellipsoidal form will filter more of the dynamics. However, even understanding the limits of such a model would be extremely revealing.

The application of the ellipsoidal form to geophysical vortex dynamics has only a short history, beginning with the works of Zhmur and Pankratov [8, 9] and independently Meacham [10]. Despite this relatively short period, it already has yielded many insights into vortex interactions in geophysical flows. These vortex interactions are crucial as they are a huge driving force within the atmospheric and the oceanic circulation. There is still great potential for this subject to reveal more about the complex dynamics within geophysical turbulence.

\section{Conflict of Interests}

The author declares that there is no conflict of interests regarding the publication of this paper.

\section{Acknowledgments}

This work has been funded by the Italian Flagship Project RITMARE, The Italian Research for the Sea, coordinated by the Italian National Research Council and funded by the Italian Ministry of Education, University and Research.

\section{References}

[1] D. B. Chelton, M. G. Schlax, and R. M. Samelson, "Global observations of nonlinear mesoscale eddies," Progress in Oceanography, vol. 91, no. 2, pp. 167-216, 2011.

[2] Z. Zhang, W. Wang, and B. Qiu, "Oceanic mass transport by mesoscale eddies," Science, vol. 345, pp. 322-324, 2014.

[3] J. C. McWilliams, J. B. Weiss, and I. Yavneh, "Anisotropy and coherent vortex structures in planetary turbulence," Science, vol. 264, no. 5157, pp. 410-413, 1994.

[4] K. Shaper Smith and G. K. Vallis, "The scales and equilibration of Midocean Eddies: freely evolving flow," Journal of Physical Oceanography, vol. 31, no. 2, pp. 554-571, 2001.

[5] W. J. Mckiver and D. G. Dritschel, "Balance in non-hydrostatic rotating stratified turbulence," Journal of Fluid Mechanics, vol. 596, pp. 201-219, 2008.

[6] G. Kirchhoff, Vorlesungen über Matematische Physic: Mechanik Teubner, Leipzig,1876.

[7] A. E. Love, "On the stability of certain vortex motions," Proceedings of the London Mathematical Society, vol. 25, pp. 18-42, 1893.

[8] V. V. Zhmur and K. K. Pankratov, "Dynamics of a semiellipsoidal subsurface vortex in a nonuniform flow," Okeanologia, vol. 29, pp. 150-154, 1989.

[9] V. V. Zhmur and K. K. Pankratov, "Dynamics of mesoscale eddy formation in the field currents of large intensive vortex," Okeanologia, vol. 30, pp. 124-129, 1990. 
[10] S. P. Meacham, "Quasigeostrophic, ellipsoidal vortices in a stratified fluid," Dynamics of Atmospheres and Oceans, vol. 16, no. 3-4, pp. 189-223, 1992.

[11] J. G. Charney, "On the scale of atmospheric motions," Geofysiske Publikasjoner, vol. 17, no. 2, pp. 3-17, 1948.

[12] C. MacClaurin, A Treatise on Fluxions, vol. 1-2, Printed by T. W. and T. Ruddimans, Edinburgh, UK, 1742.

[13] P. S. Laplace, Laplace, Pierre-Simon (1749-1827). Théorie du Mouvement et de la Figure Elliptique Des Planètes. Paris: Imprimerie de Ph.-D. Pierres, 1784.

[14] I. Todhunter, History of the Mathematical Theories of Attraction and the Figure of the Earth, Constable, London, UK, 1873.

[15] S. Chandrasekhar, Ellipsoidal Figures of Equilibrium, Dover, 1969.

[16] G. Dassios, Ellipsoidal Harmonics: Theory and Applications, Cambridge University Press, Cambridge, UK, 2012.

[17] W. J. McKiver and D. G. Dritschel, "The motion of a fluid ellipsoid in a general linear background flow," Journal of Fluid Mechanics, vol. 474, pp. 147-173, 2003.

[18] D. G. Dritschel, J. N. Reinaud, and W. J. McKiver, "The quasigeostrophic ellipsoidal vortex model," Journal of Fluid Mechanics, vol. 505, pp. 201-223, 2004.

[19] J. Pedlosky, Geophysical Fluid Dynamics, Springer, New York, NY, USA, 1979.

[20] J. G. Charney, "Geostrophic turbulence," Journal of the Atmospheric Sciences, vol. 28, no. 6, pp. 1087-1095, 1971.

[21] B. J. Hoskins, M. E. McIntyre, and A. W. Robertson, "On the use and significance of isentropic potential vorticity maps.," Quarterly Journal-Royal Meteorological Society, vol. 111, no. 470, pp. 877-946, 1985.

[22] W. R. Holland, "Quasigeostrophic modelling of Eddy-resolved ocean circulation," in Advanced Physical Oceanographic Numerical Modelling, J. J. O’Brien, Ed., vol. 186 of NATO ASI Series C: Mathematical and Physical, pp. 203-231, Springer, Dordrecht, Netherlands, 1986.

[23] B. Deremble, G. Lapeyre, and M. Ghil, "Atmospheric dynamics triggered by an oceanic SST front in a moist quasigeostrophic model," Journal of the Atmospheric Sciences, vol. 69, no. 5, pp. 1617-1632, 2012.

[24] V. V. Zhmur and A. F. Shchepetkin, "Evolution of an ellipsoidal vortex in a stratified ocean. Survivability of the vortex in a flow with vertical shear," Izvestiâ Akademii Nauk SSSR. Fizika Atmosfery i Okeana, vol. 27, pp. 492-503, 1991.

[25] R. A. Lyttleton, The Stability of Rotating Liquid Masses, Cambridge University, Paris, France, 1953.

[26] E. W. Hobson, Theory of Spherical and Ellipsoidal Harmonics, Cambridge University Press, 1931.

[27] S. P. Meacham, K. K. Pankratov, A. F. Shchepetkin, and V. V. Zhmur, "The interaction of ellipsoidal vortices with background shear flows in a stratified fluid," Dynamics of Atmospheres and Oceans, vol. 21, no. 2-3, pp. 167-212, 1994.

[28] S. P. Meacham, P. J. Morrison, and G. R. Flierl, "Hamiltonian moment reduction for describing vortices in shear," Physics of Fluids, vol. 9, no. 8, pp. 2310-2328, 1997.

[29] H. Hashimoto, T. Shimonishi, and T. Miyazaki, "Quasigeostrophic ellipsoidal vortices in a two-dimensional strain field," Journal of the Physical Society of Japan, vol. 68, no. 12, pp. 38633880, 1999.

[30] J. N. Reinaud, D. G. Dritschel, and C. R. Koudella, "The shape of vortices in quasi-geostrophic turbulence," Journal of Fluid Mechanics, no. 474, pp. 175-192, 2003.
[31] D. G. Dritschel, R. K. Scott, and J. N. Reinaud, "The stability of quasi-geostrophic ellipsoidal vortices," Journal of Fluid Mechanics, vol. 536, pp. 401-421, 2005.

[32] T. Miyazaki, K. Ueno, and T. Shimonishi, "Quasigeostrophic, tilted spheroidal vortices," Journal of the Physical Society of Japan, vol. 68, no. 8, pp. 2592-2601, 1999.

[33] W. J. McKiver and D. G. Dritschel, "The stability of a quasigeostrophic ellipsoidal vortex in a background shear flow," Journal of Fluid Mechanics, vol. 560, pp. 1-17, 2006.

[34] J. N. Reinaud and D. G. Dritschel, "The merger of vertically offset quasi-geostrophic vortices," Journal of Fluid Mechanics, vol. 469, pp. 287-315, 2002.

[35] V. V. Zhmur and K. K. Pankratov, "Distant interaction of an ensemble of quasigeostrophic ellipsoidal eddies: Hamiltonian formulation," Atmospheric and Oceanic Physics, vol. 26, pp. 972981, 1990.

[36] T. Miyazaki, Y. Furuichi, and N. Takahashi, "Quasigeostrophic ellipsoidal vortex model," Journal of the Physical Society of Japan, vol. 70, no. 7, pp. 1942-1953, 2001.

[37] Y. Li, H. Taira, N. Takahashi, and T. Miyazaki, "Refinements on the quasi-geostrophic ellipsoidal vortex model," Physics of Fluids, vol. 18, no. 7, Article ID 076604, 2006.

[38] N. Martinsen-Burrell, K. Julien, M. R. Petersen, and J. B. Weiss, "Merger and alignment in a reduced model for threedimensional quasigeostrophic ellipsoidal vortices," Physics of Fluids, vol. 18, no. 5, Article ID 057101, 2006.

[39] J. N. Reinaud and D. G. Dritschel, "The critical merger distance between two co-rotating quasi-geostrophic vortices," Journal of Fluid Mechanics, vol. 522, pp. 357-381, 2005.

[40] T. Miyazaki, M. Yamamoto, and S. Fujishima, "Counter-rotating quasigeostrophic ellipsoidal vortex pair," Journal of the Physical Society of Japan, vol. 72, no. 8, pp. 1948-1962, 2003.

[41] K. V. Koshel, E. A. Ryzhov, and V. V. Zhmur, "Diffusion-affected passive scalar transport in an ellipsoidal vortex in a shear flow," Nonlinear Processes in Geophysics, vol. 20, no. 4, pp. 437-444, 2013.

[42] Y.-K. Tsang and D. G. Dritschel, "Ellipsoidal vortices in rotating stratified fluids: beyond the quasi-geostrophic approximation," Journal of Fluid Mechanics, vol. 762, pp. 196-231, 2015.

[43] W. J. McKiver and D. G. Dritschel, "Balanced solutions for an ellipsoidal vortex in a rotating stratified flow," Journal of Fluid Mechanics, submitted. 


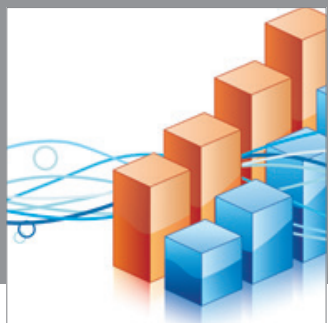

Advances in

Operations Research

mansans

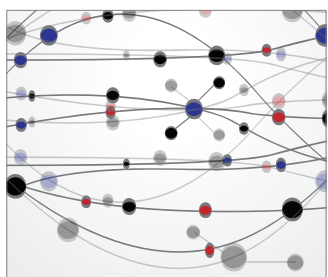

The Scientific World Journal
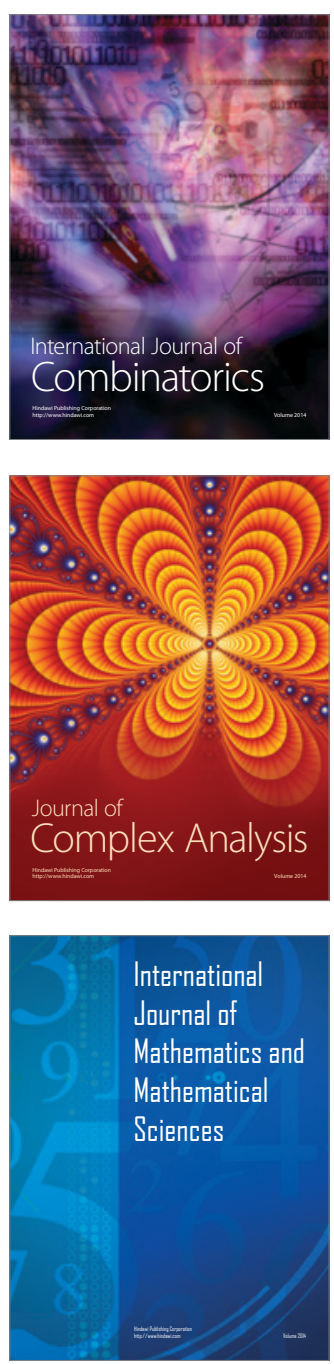
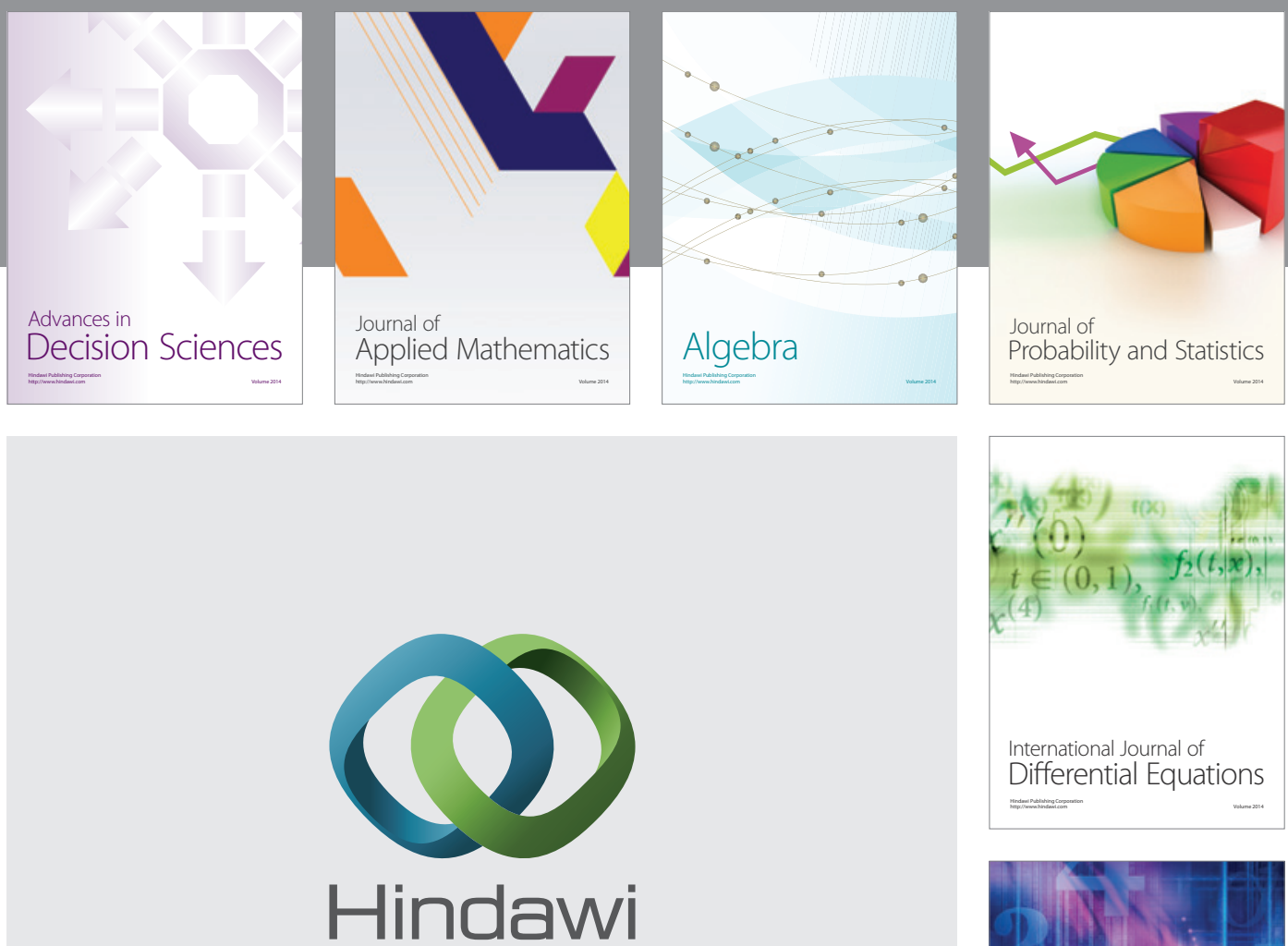

Submit your manuscripts at http://www.hindawi.com
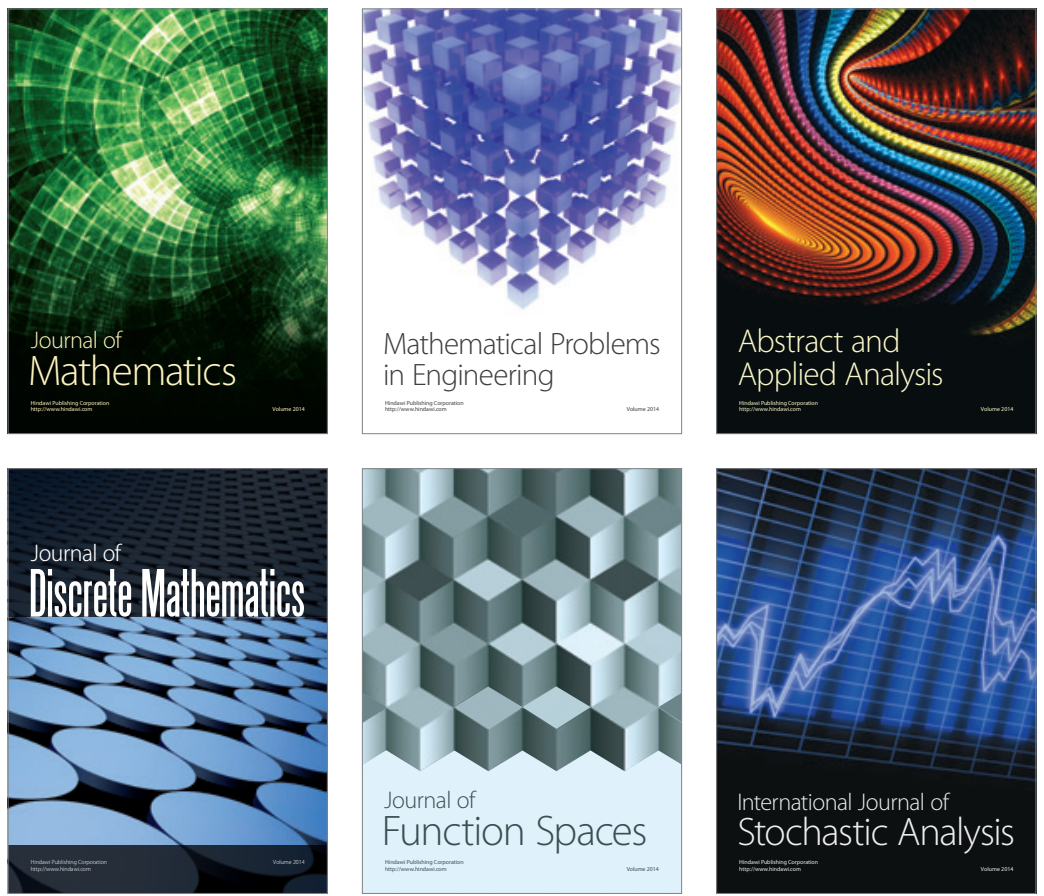

Journal of

Function Spaces

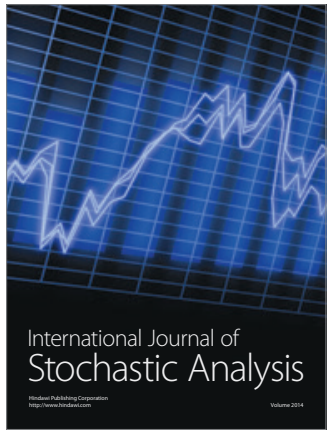

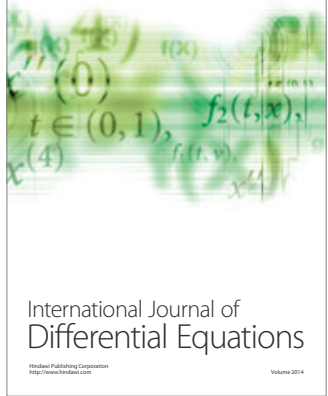
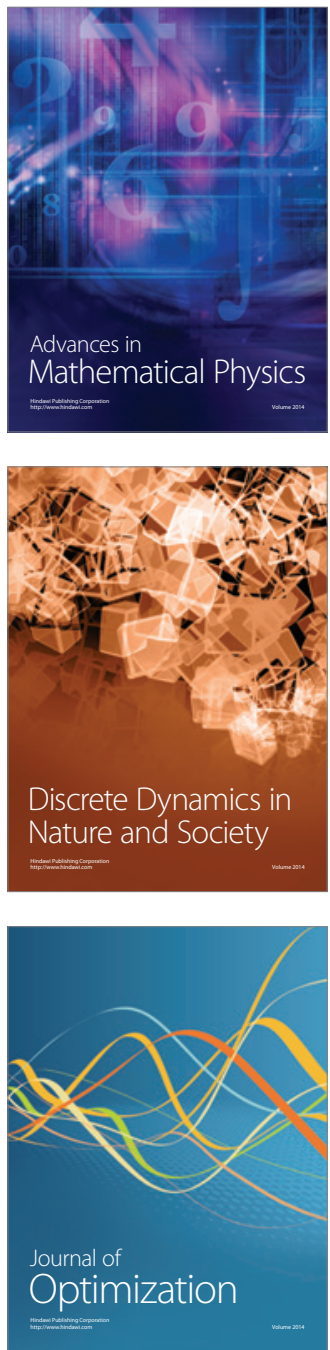